Preprint of: Cetó Alsedà, Xavier et al. "Determination of total polyphenol index in wines employing a voltammetric electronic tongue" in Analytica chimica acta, available online Vol. 732 (Jun 2012), p. 172-179.

The final version is available at 10.1016/j.aca.2012.02.026

1 Determination of total polyphenol index in wines employing a

\title{
voltammetric Electronic Tongue
}

\author{
Xavier Cetó ${ }^{1}$, Juan Manuel Gutiérrez ${ }^{2}$, Manuel Gutiérrez ${ }^{3}$, Francisco Céspedes ${ }^{1}$, \\ Josefina Capdevila ${ }^{4}$, Santiago Mínguez ${ }^{4}$, Cecilia Jiménez-Jorquera ${ }^{3}$ and Manel del \\ Valle $e^{1, *}$ \\ ${ }^{1}$ Sensors and Biosensors Group, Department of Chemistry, Universitat Autònoma de \\ Barcelona, Edifici Cn, 08193 Bellaterra, SPAIN \\ ${ }^{2}$ Bioelectronics Section, Department of Electrical Engineering, CINVESTAV, 07360 \\ Mexico D.F., Mexico \\ ${ }^{3}$ Instituto de Microelectronica de Barcelona (IMB-CNM), CSIC, 08193 Bellaterra, \\ Spain \\ ${ }^{4}$ Estació de Viticultura i Enologia, INCAVI, Vilafranca del Penedes, Spain
}

\section{Abstract}

This work reports the application of a voltammetric Electronic Tongue system (ET) made from an array of modified graphite-epoxy composites plus a gold microelectrode in the qualitative and quantitative analysis of polyphenols found in wine. Wine samples were analyzed using cyclic voltammetry without any sample pretreatment. The obtained responses were preprocessed employing Discrete Wavelet Transform in order to compress and extract significant features from the voltammetric signals, and the obtained approximation coefficients fed two multivariate calibration methods (ANN and PLS) which accomplish the quantification of total polyphenol content. External test results were compared with the ones obtained with the Folin-Ciocalteu method and UV absorbance polyphenol index $\left(\mathrm{I}_{280}\right)$ as reference values, with highly significant correlation coefficients of 0.979 and 0.963 in the range from 50 to $2400 \mathrm{mg} \mathrm{L}^{-1}$ gallic acid equivalents, respectively. In a separate experiment, qualitative discrimination of different polyphenols found in wine was also assessed by Principal Component Analysis.

Keywords: voltammetric sensors; electronic tongue; Artificial Neural Network; wine analysis; Folin-Ciocalteu; polyphenol

\footnotetext{
*E-mail: manel.delvalle@uab.cat; tel: +34 93 5811017; fax: +34 935812379
} 


\section{Introduction}

Wine is an important analytical field, taking special attention to new methodologies for its characterization and elaboration control [1]. One important wine parameter, determining some organoleptic and sensorial properties, is its polyphenol content [2]. Phenolics in plants may act as phytoalexins, antifeedants, attractants for pollinators, contributors to plant pigmentation, antioxidants and protective agents against UV light, among others [3]. Meanwhile in food, phenolics may contribute to the bitterness, astringency, color, flavor, odor and oxidative stability of food. In addition to health-protecting capacity and some properties other than nutritional of plants, phenolics are of great importance to both consumers and producers.

In grapes, synthesis of polyphenols is induced by factors such as grape cultivar, developmental stage of the berry and maturation, climatology and UV radiation and viticultural practices; it can also be affected by fungal infection (Botrytis cinerea) and injuries [4-6]. In wine, phenolics are responsible of pigmentation (in red but also in white wines), aging, oxygen-depleting compounds and bitter and stringent components, which are determinant for the wine taste and character.

Several methods to quantify total phenols and polyphenols have been described in the literature [7]. The Folin-Ciocalteu (FC) method is widely employed in the wine industry [8]. This spectrophotometric method measures the sample reducing capacity. As a faster alternative to this method, the use of what is known as polyphenol index $\left(\mathrm{I}_{280}\right)$ is now arising [9]; in this case wine absorbance is measured directly at $280 \mathrm{~nm}$ and straightly correlated with phenolic content. Another spectrophotometric method widely used is the reaction with 4-aminoantypirine [10], a generic reaction for phenols. On the one hand, these methods yield a total phenol content value, and therefore do not allow for the discrimination between individual constituents. However there are some spectrophotometric methods, developed for quantification of phenolics in plants, that brings some specificity; these assays are based on differential reactivity principles and are used to determine different structural groups concominant in phenolic compounds [11]. On the other hand, there is also the use of chromatographic techniques such as HPLC [12] or GC [13], which are able to perform individual determination of phenolic compounds; however these methods need complex and time-consuming sample pretreatment procedures, and are not suitable for on-site analyses. Nowadays, biosensors are arising as an alternative to the traditional techniques given their low cost and their 
ease of use to carry out on field analyses. Therefore, biosensors represent an attractive alternative also for the detection of polyphenolic compounds. For this aim, biosensors have been developed incorporating enzymes such as Laccase [14], Tyrosinase [15] or Peroxidase [16], and even by the coimmobilization of two enzymes in the same biosensor [17].

The present work reports the application of an Electronic Tongue (ET) in the analysis of polyphenols. The ET is a recent trend from the sensory field, which entails the use of an array of sensors capable of giving a wide and complete response of the analyzed species, plus a chemometric processing tool able to interpret the chemical signals and extract meaningful data from the complex readings [18, 19]. Although there are only a few number of papers related to ETs and wine, they have been previously applied to distinguish different wine varieties and tastes [20-22], to quantify some analytical parameters of wines [23-25] or even to detect wine adulterations [26, 27]. Potentiometric sensors have been used as well, and their signal correlated to polyphenol content of wines [28].

The ET is, in this sense, the hybrid formed between sensors and the use of chemometrics, such as Artificial Neural Networks (ANNs); these advanced signal processing variants allows the interpretation, modelling and calibration of complex analytical signals $[29,30]$. Despite great advantages provided by the use of ANNs, when voltammetric sensors are used, the high complexity of the generated data matrix hinders their treatment. The straightforward solution is the use of multiway processing methods (samples $\mathrm{x}$ sensors $\mathrm{x}$ polarization potential), but the complexity of this technique is also critical $[31,32]$. One solution when dealing with a set of voltammograms is to employ a preprocessing stage for data reduction; this option permits to gain advantages in training time, to avoid redundancy in input data and to obtain a model with better generalization ability. This compression stage may be achieved by the use of methods such as Principal Component Analysis (PCA), "kernels" [33] or Discrete Wavelet Transform (DWT) [34]. In this sense the last one, is particularly interesting because of its ability to compress and denoise data.

Here we report a multidimensional ET aimed for the determination of the total polyphenol content, formed by an array of six voltammetric sensors: a Au microelectrode plus five epoxy-graphite composite sensors, four of them bulk-modified with cobalt phtalocyanine, different nanoparticles $(\mathrm{Pt}$ and $\mathrm{Cu})$ and polypyrrole powder (a conducting polymer). This array is designed in this manner to produce differentiated 
catalytic responses to specific phenolic compounds present in the wines [27]. Obtained responses were preprocessed employing DWT in order to extract the significant information present and the resulting approximation coefficients fed the different multivariate calibration methods (ANN and PLS-2 models), specially trained to predict the total polyphenol indexes, in what might be considered a bioinspired analytical method that uses artificial intelligence tools.

\section{Experimental}

\subsection{Samples under study}

A total of 20 wine samples of different varieties from 2008 and 2009 vintage were analyzed; all of them were from the Penedès region (Catalonia, Spain). Samples were selected as to obtain a set with sufficiently differentiated total polyphenol indexes and grape varieties, with values ranged from 54 to $2374 \mathrm{mg} \mathrm{L}^{-1}$ gallic acid equivalents.

$<$ TABLE $1>$

\subsection{Reagents and solutions}

All reagents used were analytical reagent grade and all solutions were prepared using deionised water from a Milli-Q system (Millipore, Billerica, MA, USA). Gallic acid, catechin, $p$-coumaric acid, caffeic acid, catechol, $m$-cresol, copper and platinum nanoparticles $(<50 \mathrm{~nm})$, polynailine and polypyrrole were purchased from SigmaAldrich (St. Louis, MO, USA). KCl was purchased from Merck KGaA (Darmstadt,

125 Germany). Folin-Ciocalteu's Reagent and Sodium Carbonate were purchased from Panreac Química (Barcelona, Spain). A solution containing 0.1 $\mathrm{M} \mathrm{KNO}_{3}$ (Fluka) was used to activate the gold $(\mathrm{Au})$ microelectrode.

\subsection{Determination of polyphenol content by Folin-Ciocalteu method and} spectrophotometric measurements

For comparison purposes, Folin-Ciocalteau (FC) index of the wines was also analyzed spectrophotometrically [35]. The FC test was carried out according to the 
133 following procedure: $1 \mathrm{~mL}$ of sample (wines were diluted 1:100 or 1:50), $6 \mathrm{~mL}$ of 134 deionized water, $0.5 \mathrm{~mL}$ of Folin-Ciocalteu reagent and $2 \mathrm{~mL}$ of a $20 \%$ sodium 135 carbonate solution were added in this order to a $10 \mathrm{~mL}$ beaker and diluted to volume 136 with deionized water. The resulting solution was stirred and allowed to react for half an 137 hour at room temperature in darkness. The absorbance was then read at $760 \mathrm{~nm}$ by a 138 spectrophotometer Perkin Elmer Lambda 20 UV/VIS. Total phenolic content, expressed 139 in gallic acid equivalents, was evaluated from the absorbance value by interpolation into 140 the calibration plot obtained with gallic acid standard solutions, multiplying the 141 resulting value by 10 and by the proper dilution rate. Different dilution factors were 142 applied given when carrying out specthrophotometric measurements absorbance must 143 be around 0.3 [35].

144 For the determination of the total content of polyphenolic compounds in wines 145 the polyphenol index $\mathrm{I}_{280}$ was also considered: wine was diluted with water (1:100 or $146 \mathrm{1}: 50$ ) and the absorbance was measured directly at $280 \mathrm{~nm}$. The value of $\mathrm{I}_{280}$ for each 147 sample was given as the absorbance multiplied by the proper dilution rate. In both 148 methods the blank solution was a hydro-alcoholic solution $(12 \%$, v/v ethanol) of tartaric acid $3 \mathrm{~g} / \mathrm{L}$.

\subsection{Electrochemical measurements}

152 Samples were analysed with two types of amperometric sensors: an array of 5 153 graphite-epoxy voltammetric sensors made with different modifiers added to the bulk 154 mixture, selected according with previous experiments in our laboratory [27, 36], and a 155 microfabricated Au microelectrode [37, 38]. Electroanalytical experiments were carried 156 out at room temperature $\left(25^{\circ} \mathrm{C}\right)$ under quiescent conditions, and without any pre157 treatment or dilution of the sample. Electrodes were cycled in saline solution in order to 158 get stable voltammetric responses before performing the measurements with real 159 samples.

\subsubsection{Composite electrodes}

Working electrodes were prepared following the conventional methodology previously established in our research Group [39]. A resin EpoTek H77 (Epoxy

164 Technology, Billerica, MA, USA) and its corresponding hardener compound were 165 mixed in the ratio $20: 3(\mathrm{w} / \mathrm{w})$; afterwards a $15 \%$ of graphite $(\mathrm{w} / \mathrm{w})$ and a $2 \%$ of the 166 modifier $(\mathrm{w} / \mathrm{w})$ were added to the previous mixture before hardening, obtaining the 
167 composite. Then, it was manually homogenized for $60 \mathrm{~min}$, and afterwards composite 168 paste was allowed to harden during 3 days at $80{ }^{\circ} \mathrm{C}$. Finally, electrode surface was 169 polished with different sandpapers of decreasing grain size, with a final electrode area 170 of $28 \mathrm{~mm}^{2}$

171 In this manner, an array of 5 different graphite-epoxy voltammetric sensors were 172 prepared using bare graphite $\mathrm{C}$, adding different modifiers such as cobalt phtalocyanine, 173 conducting polymer as polypyrrole and nanoparticles of copper and platinum to the bulk 174 mixture - one component per electrode.

\subsubsection{Gold microelectrode}

177 Also a conventional $\mathrm{Au}$ microelectrode fabricated according to standard 178 photolithographic techniques was used [40]. The $\mathrm{Au}$ microelectrode was firstly 179 chemically cleaned successively with ethanol $96 \%, \mathrm{H}_{2} \mathrm{SO}_{4} 6.0 \mathrm{M}$ and de-ionized water.

180 Next, an electrochemical activation was carried out in $0.1 \mathrm{M} \mathrm{KNO}_{3}$, where the electrode was cycled from +0.8 to $-2.2 \mathrm{~V}$ for at least 20 times.

\subsubsection{Amperometric measurements}

184 Amperometric measurement cell was formed by the 6-sensor voltammetric array and a reference double junction $\mathrm{Ag} / \mathrm{AgCl}$ electrode (Thermo Orion 900200, Beverly, MA, USA) plus a commercial platinum counter electrode (Model 52-67, Crison Instruments, Barcelona, Spain). Cyclic Voltammetry measurements were taken using a 6-channel AUTOLAB PGSTAT20 (Ecochemie, Netherlands), in a multichannel configuration, using GPES Multichannel 4.7 software package. for the Au microelectrode), with a scan rate of $100 \mathrm{mV} \mathrm{s}^{-1}$ and a step potential of $9 \mathrm{mV}$. Apart, all experiments were carried out without performing any physical surface regeneration of the working electrodes. In order to prevent the accumulative effect of impurities on the working electrode surfaces, an electrochemical cleaning stage was done between each measurement applying a conditioning potential of $+1.5 \mathrm{~V}$ during 40 $\mathrm{s}$ after each experiment, in a cell containing $25 \mathrm{ml}$ of distilled water [36].

\subsection{Spiked samples}

Beyond wine samples summarized in Table 1, some spiked samples were prepared in order to assess the discrimination ability shown by the sensor array. For 
this, a reference wine was spiked with different quantities of a stock solution of certain polyphenols. Polyphenols considered were selected according to major polyphenols found in wine [41, 42]; gallic acid, catechin, $p$-coumaric acid, caffeic acid, catechol and $m$-Cresol were the ones tested. It must be reckoned that average total polyphenol content measured by the Folin method is higher than the individual content of each polyphenol [41, 43]. For example, total polyphenol content is around $2160 \mathrm{mg} \mathrm{L}^{-1}$ for red wine, $820 \mathrm{mg} \mathrm{L}^{-1}$ in rosé wine and $320 \mathrm{mg} \mathrm{L}^{-1}$ for white wines; however individual polyphenol concentration is much lower with values under $300 \mathrm{mg} \mathrm{L}^{-1}$ for red wine and $50 \mathrm{mg} \mathrm{L}^{-1}$ for white wines [43].

In this manner, a rosé wine was choosen as the one to which phenolic compounds will be added given their lower polyphenolic content. Then, from it, 35

212 different samples were prepared based on 7 groups (one for each of the 6 polyphenols 213 under study plus another for the unspiked wine). To confirm that differentiation 214 between compounds was not due to different amount of phenolic compounds being 215 added, in all the cases the same amount was added. Thus, for each class $5 \mu$ mols of 216 polyphenolic compound were added to $25 \mathrm{~mL}$ of wine, which represents approximately 217 an increase of $36 \mathrm{mg} \mathrm{L}^{-1}(200 \mu \mathrm{M})$.

\subsection{Data processing}

Chemometric processing was done by specific routines in MATLAB 7.0 (MathWorks, Natick, MA) written by the authors, using Neural Network and Wavelet Toolboxes (v.4.0). Partial Least Squares (PLS) regression was done employing The Unscrambler (CAMO Software AS, Oslo, Norway) informatics package. Sigmaplot 2000 (Systat Software Inc, California, USA) was used for graphic representations of data and results.

The whole cyclic voltammograms obtained from each sensor were included in the data processing stage. In order to reduce the multidimensional data matrix generated in each measurement, a preprocessing stage employing the Discrete Wavelet Transform (DWT) was used [44]. In this way, and using the proposed sensor array, the corresponding compressed voltammograms were processed employing either an

231 Artificial Neural Network (ANN) or Partial Least Squares (PLS-2). Both models allowed carrying out the quantification of the FC index and the $\mathrm{I}_{280}$ values. optimize the configuration details that determine its operation. Normally, this is a trial- 
and-error process, where several parameters (training algorithms, number of hidden

236 layers, transfer functions, etc.) are fine-tuned in order to find the best configuration to optimize the performance of the model.

Given the complexity of the data set, also a linear model like PLS-2 was evaluated; PLS-2 was chosen given its ability to model several variables together, unlike PLS-1 or PCR. Therefore obtained model would be equivalent to the one built with ANN. In this case, the parameter that must be taken into account are the number of PCs used to build the PLS-2 model; to optimize this parameter, it was taken into account the model that gives the lower total Normalized Root Mean Square Error (NRMSE) for predicted values. Also, as done in the case of ANN voltammetric signals were preprocessed employing DWT before adjusting PLS, given this data reduction improves model prediction and generalization abilities [45].

Given the reduced size of the data set, a jack-knife method was used [46, 47]. Jack-knife method was suggested for use in statistics to describe a general approach for testing hypotheses and calculating confidence intervals in situations where apparently no better methods can be used. With this procedure, standard errors are calculated from different resampling with random distribution and repeating the modelling stage. In this manner, it could be used either leaving one sample out each timer or even many samples out each time [47].

In our case, it was applied using 15 samples for training process and leaving 5 samples out each time which were used to evaluate model's response; then, this subdivision of the original data set was repeated 25 times leaving 5 different samples out each time, which were selected randomly. Once all the responses from the models were obtained, data was grouped depending if it was intervening in the training process or used as external test.

Also Principal Component Analysis (PCA) was used to visualize the discrimination capability derived from the voltammetric signals acquired, and combined with cluster analysis tools to build a preliminary recognition model. PCA allows to project the information carried by the original variables onto a smaller number of underlying ("latent") variables called principal components (PCs) with new coordinates called scores, obtained after data transformation. Then by plotting the PCs, one can view interrelationships between different variables, and detect and interpret sample patterns, groupings, similarities or differences [27]. Moreover, PCA is a useful method to reduce the dimensionality of large data sets, such as those from arrays of 
voltammetric sensors. Cluster analysis tools allow constructing clusters from data using pairwise distance between observations (usually Euclidean distance), as linkage to construct the hierarchical cluster tree.

\section{Results and Discussion}

\subsection{Voltammetric responses and feature extraction}

276 Different voltammetric responses are observed for each kind of sensor, as shown

277 in Figure 1. These signals presumably contribute in different manner with the data

278 needed for model quantification. Differentiated signals are obtained for each type of 279 sensor used. Catalytic oxidative signals seem to originate from the sensors using metal 280 nanoparticles, which may be due to a catalytic oxidation of saccharides and/or 281 polyphenols. Similarly, sensors modified with conducting polymers bring new 282 information with completely different waveforms. Gold electrode would provide the 283 most generic redox behaviour of the sample. At the same time, it can be seen how 284 currents increase in the same way as FC index increases and with different behaviour 285 for each sensor; meanwhile each sensor shows its distinctive profile, generating very 286 rich data that is very useful as departure point. For developing an ET, the first necessary 287 condition is to have analytical signals responding to the phenomena to which the objective is aimed, with variability among them and the different sensors forming the sensor array. As demonstrated, bulk modification of voltammetric electrodes is an easy way to enrich the cross-response of the sensor array to different aspects of the solution under study [27]. Even the extreme complexity of the generated signals (the set of voltammograms) hinders the processing step. As already commented, all these data is used performing a compression step, required to gain advantages in training time, to avoid redundancy in input data and to obtain a model with better generalization ability. An additional reason is the need to extract reduced and significant information compatible with the ANN structure [44]. This was accomplished by use of the DWT. 
A DWT preprocessing stage was performed employing Daubechies wavelet and a sixth decomposition level, the best choice from preliminary tests and previous experience $[34,44]$. The DWT allowed compressing the original data set information up to $97.27 \%$ without any loss of relevant information. From the proposed 6-sensor array, the corresponding voltammograms were compressed, and the obtained coefficients were fed into multivariate calibration methods in order to predict the total polyphenol content in wines. In this sense, two different methods were evaluated: an ANN as a non-linear data modelling tool and PLS-2 as a linear one. A simplified scheme of the procedure followed for data treatment and ANN architecture can be observed in Figure 2.

310

$<$ FIGURE 2>

After a systematic evaluation of topologies, the final DWT-ANN architecture model had 84 input neurons (corresponding to the 14 wavelet approximation coefficients obtained from wavelet analysis of each of the 6 sensor signals), 10 neurons and logsig function in the hidden layer and 2 output neurons and purelin function in the output layer corresponding to $\mathrm{FC}$ and $\mathrm{I}_{280}$ indexes. Although, individual ANN models to predict separately these two indexes may also be developed. Bayesian regularization was used to train the network, this algorithm has the advantage of avoiding overfitting without the need of an internal validation subset [44], then this precaution is not performed.

For the optimization of PLS model only two considerations were taken into account: the differences obtained when using the 84 DWT coefficients instead of the raw voltammetric data and the number of PCs used to build the model. Despite PLS has no need of a preprocessing stage, it was found that better models were obtained when this was performed (total NRMSE improved from 0.31 to 0.20 for predicted values). Thus, the final model was a DWT-PLS2 with 7 PCs, which has a total explained variance ca. $95.2 \%$.

After building the models, which were evaluated for training with $75 \%$ of the data and tested with the remaining $25 \%$, a jack-knife method was employed to visualize dependence of predictions from the specific subdivision of data. Then, in order to characterize the accuracy of the identification models and obtain unbiased data, train/test data division was repeated randomly 25 times using a 5-fold cross validation 
334 process; this precaution ensured that performance indicators were independent of the 335 subsets used.

336 Comparison graphs for each model were built grouping the replicas for each 337 individual sample, differentiating when it was intervening in the training process and 338 when used as external test. The predicted indexes were then plot against the expected 339 ones and fitted with linear least-squares regression. To give the same weight to all 340 points, weighed regression was used. The obtained results for the ANN model can be 341 seen on Figure 3 for the Folin-Ciocalteu Index and Figure 4, for the $\mathrm{I}_{280}$ index. In the 342 same way, results from the DWT-PLS2 model can be seen in Figure 5 for the FC index, 343 and Figure 6 for the $I_{280}$ index. In both cases, an estimation of prediction errors 344 (intervals calculated at the 95\% confidence level) corresponding to the average of the 25 345 jack-knife calculations, taken from the dispersion of the replicas, can be also visualized.

$<$ FIGURE 3>

$348<$ FIGURE 4>

$349<$ FIGURE 5>

$<$ FIGURE 6>

As usual in multivariate calibration methods work, training stage behaviour shows better agreement and reduced errors than testing stage. The general behaviour is also good in both cases, although individual prediction errors are largely increased, especially for certain samples that were more dependant on the specific data used in training. Despite the good trend in both cases, larger uncertainties were obtained with the DWT-PLS2, thus meaning ANN model is slightly more robust. Regardless of this, Table 2 presents the results of the weighed regressions, where the good correlation coefficients and the small associated errors stand out (intervals calculated at the $95 \%$ confidence level). The obtained comparison results are close to the ideal values, with intercepts near to 0 and slopes and correlation coefficients around 1, meaning that there are no significant differences between the values predicted by the multivariate calibration methods and the reference ones.

$<$ TABLE 2> 
Employing the same ET and following equivalent procedure for the data treatment, spiked wine samples with typical polyphenolic compounds present in wine, as described in Section 2.5, were measured as before but in random order. An extract of the results is shown on Figure 7, corresponding to the polypyrrole sensor; which was selected for being the one where the most clearly differentiated cathodic response is obtained for each compound. Then the information obtained from the complete set of voltammograms was evaluated using PCA and groups were formed using cluster analysis tools.

$<$ FIGURE 7>

The PCA analysis was done, and with the two first PCs, the explained variance accumulated was ca. $95 \%$; a large value which means that nearly all the variance contained in the original data is explained. By plotting them, different clusters were obtained as can be seen in Figure 8; patterns in the figure evidence that wine samples are grouped based on which polyphenol was added. These well established clusters clearly separate the main classes of samples corresponding to: (I) Wine, (II) Gallic acid, (III) Catechin, (IV) $p$-Coumaric acid, (V) Caffeic acid, (VI) Catechol and (VII) $m$ Cresol.

$<$ FIGURE 8 $>$

The fact that gallic acid and catechin clusters were so close one to the other, and also from the wine cluster, is due to the fact that these two compounds are the ones present in higher concentration in wine. As expected, $p$-Coumaric and caffeic acids clusters were more separated to the wine cluster and close between them, given both are hydroxycinnamic acids differing only by one alcohol group. Finally, $m$-Cresol and

395 Catechol were clearly far away from the rest of clusters and mainly represented by PC2.

396 The position of these clusters could be explained by the fact that these components are 397 found in less proportion in wine and their chemical structure is quite different from the others.

In this interpretation of polyphenol identification, we could see that individual groups were obtained for each phenolic compound; moreover, compounds with structural similarity arranged closely between them, meaning there is some background 
relationship. Although the good results obtained with those spiked samples, it should be taken into account that the quantification of individual polyphenols in a complex sample such as wine is very difficult, but the results obtained suggest this possibility. In essence, the study demonstrates the capabilities of the proposed ET, both in the quantification of total polyphenol content and in the differentiation of different polyphenols found in wine.

408

\section{Conclusions}

An electronic tongue based on voltammetric sensors with different modifiers

412 (metallic nano-sized particles, conducting polymers and cobalt phtalocyanine) was

413 developed in order to create a tool capable of quantifying total polyphenol content in

414 wines. Complex voltammetric data required the use of a preprocessing stage that was

415 achieved by the use of DWT, which provides a good compression of data preserving 416 relevant information. While the use of ANN or PLS-2 allowed predicting phenolic 417 content indexes obtained with two different reference methods (Folin-Ciocalteu and $\mathrm{I}_{280}$ 418 indexes). In a more qualitative application of the technique, we have been also able to 419 differentiate between different polyphenols found in wine samples by the use of PCA, 420 clearly discriminating them in an application comparable to much more complex 421 analytical techniques such as HPLC.

422 Finally, superior performance of ET combined with the use of chemometric 423 tools as ANN in tasks multivariate calibration or pattern recognition has been again 424 demonstrated, presenting the proposed ET as an alternative to traditional methods for 425 polyphenols quantification and even distinction of different polyphenols present in 426 wine.

\section{Acknowledgments}

Financial support for this work was provided by Spanish Ministry of Science and 431 Innovation MCINN (Madrid) trough the projects TEC2007-68012-C03 and CTQ2010432 17099, by the Mexican National Council of Science and Technology CONACYT 433 (Mexico) through the project 154243 and by the Catalonian program ICREA Academia. 
434 X. Cetó thanks the support of Dept. d'Innovació, Universitats i Empresa de la 435 Generalitat de Catalunya for the predoctoral grant.

436 
439 [1] I.S. Arvanitoyannis, M.N. Katsota, E.P. Psarra, E.H. Soufleros, S. Kallithraka, 440 Trends Food Sci. Tech., 10 (1999) 321.

441 [2] P. Ribéreau-Gayon, Y. Glories, A. Maujean, D. Dubourdieu, Handbook of 442 Enology, John Wiley \& Sons, Ltd, 2006, p. 141.

443 [3] F. Shahidi, M. Naczk, Phenolics in Food and Nutraceuticals: Sources, 444 Applications and Health Effects, CRC Press, FL, 2003.

445 [4] L. Bavaresco, D. Petegolli, E. Cantù, M. Fregoni, G. Chiusa, M. Trevisan, Vitis, $44636(1997) 77$.

447 [5] A. Versari, G.P. Parpinello, G.B. Tornielli, R. Ferrarini, C. Giulivo, J. Agr. Food 448 Chem., 49 (2001) 5531.

449 [6] E.H. Siemann, L.L. Creasy, Am. J. Enol. Vitic., 43 (1992) 49.

450 [7] I. Ignat, I. Volf, V.I. Popa, Food Chem., 126 (2011) 1821.

451 [8] V.L. Singleton, J.A. Rossi, Amer J Enol Viticult, 16 (1965) 144.

452 [9] E. Mataix, M.D.L. de Castro, Analyst, 126 (2001) 251.

453 [10] M. Fayyad, M. Tutunji, L. Abdel-Nour, Dirasat Nat. Sci., 10 (1983) 107.

454 [11] M. Naczk, F. Shahidi, J. Pharmaceut. Biomed., 41 (2006) 1523.

455 [12] H. Sakakibara, Y. Honda, S. Nakagawa, H. Ashida, K. Kanazawa, J. Agr. Food 456 Chem., 51 (2002) 571.

457 [13] G.J. Soleas, E.P. Diamandis, A. Karumanchiri, D.M. Goldberg, Anal. Chem., 69 458 (1997) 4405.

459 [14] D.M.A. Gil, M.J.F. Rebelo, Eur. Food Res. Technol., 231 (2010) 303.

460 [15] V.C. Sanz, M.L. Mena, A. Gonzalez-Cortes, P. Yanez-Sedeno, J.M. Pingarron, 461 Anal. Chim. Acta, 528 (2005) 1.

462 [16] R. Solna, P. Skladal, Electroanal., 17 (2005) 2137.

463 [17] M.R. Montereali, L. Della Seta, W. Vastarella, R. Pilloton, J. Mol. Catal. B464 Enzym., 64 (2010) 189.

465 [18] M. del Valle, Electroanal., 22 (2010) 1539.

466 [19] Y. Vlasov, A. Legin, A. Rudnitskaya, C. Di Natale, A. D'Amico, Pure Appl. 467 Chem., 77 (2005) 1965.

468 [20] M.L. Rodríguez-Méndez, V. Parra, C. Apetrei, S. Villanueva, M. Gay, N. Prieto, 469 J. Martinez, J.A. de Saja, Microchim. Acta, 163 (2008) 23.

470 [21] S. Buratti, S. Benedetti, M. Scampicchio, E.C. Pangerod, Anal. Chim. Acta, 525 471 (2004) 133.

472 [22] L. Pigani, G. Foca, K. Ionescu, V. Martina, A. Ulrici, F. Terzi, M. Vignali, C. 473 Zanardi, R. Seeber, Anal. Chim. Acta, 614 (2008) 213.

474 [23] A. Legin, A. Rudnitskaya, L. Lvova, Y. Vlasov, C. Di Natale, A. D'Amico, 475 Anal. Chim. Acta, 484 (2003) 33.

476 [24] M. Gay, C. Apetrei, I. Nevares, M. del Alamo, J. Zurro, N. Prieto, J.A. De Saja, 477 M.L. Rodriguez-Mendez, Electrochim. Acta, 55 (2010) 6782.

[25] X. Cetó, F. Céspedes, M.I. Pividori, J.M. Gutiérrez, M. del Valle, Analyst (2012). doi: 10.1039/c1an15456g

[26] V. Parra, Á.A. Arrieta, J.-A. Fernández-Escudero, M.L. Rodríguez-Méndez, J.A. De Saja, Sensor Actuat. B-Chem., 118 (2006) 448. Microchim. Acta, 169 (2010) 261.

[28] G. Verrelli, L. Lvova, R. Paolesse, C. Di Natale, A. D'Amico, Sensors, 7 (2007) 2750 . 
[29] F. Despagne, D. Luc Massart, Analyst, 123 (1998) 157R.

487 [30] Y.N. Ni, S. Kokot, Anal. Chim. Acta, 626 (2008) 130.

488 [31] D. Ebrahimi, E. Chow, J.J. Gooding, D.B. Hibbert, Analyst, 133 (2008) 1090.

489 [32] R. Cartas, A. Mimendia, A. Legin, M. del Valle, Electroanal., 23 (2011) 953.

490 [33] R. Gutiérrez-Osuna, H.T. Nagle, IEEE Sys. Man Cybern., 29 (1999) 626.

491 [34] L. Moreno-Barón, R. Cartas, A. Merkoçi, S. Alegret, M. del Valle, L. Leija, P.R. 492 Hernandez, R. Muñoz, Sensor Actuat. B-Chem., 113 (2006) 487.

493 [35] Commission Regulation (EEC), Official Journal L 272, Publication Office of the 494 European Union, 17 September 1990, p. 0001

495 [36] X. Cetó, J.M. Gutiérrez, L. Moreno-Barón, S. Alegret, M. del Valle, 496 Electroanal., 23 (2011) 72.

497 [37] M. Gutiérrez, A. Llobera, J. Vila-Planas, F. Capdevila, S. Demming, S. 498 Buttgenbach, S. Mínguez, C. Jiménez-Jorquera, Analyst, 135 (2010) 1718.

499 [38] M. Gutiérrez, C. Domingo, J. Vila-Planas, A. Ipatov, F. Capdevila, S. Demming, 500 S. Buttgenbach, A. Llobera, C. Jiménez-Jorquera, Sensor Actuat. B-Chem., 156 (2011) 501695.

502 [39] F. Céspedes, E. Martinez-Fàbregas, S. Alegret, TrAC-Trend. Anal. Chem., 15 503 (1996) 296.

504 [40] J. Orozco, G. Suarez, C. Fernandez-Sanchez, C. McNeil, C. Jimenez-Jorquera, 505 Electrochim. Acta, 53 (2007) 729.

506 [41] V. Rastija, G. Srecnik, S. Marica Medic, Food Chem., 115 (2009) 54.

507 [42] N. Paixao, R. Perestrelo, J.C. Marques, J.S. Camara, Food Chem., 105 (2007)

508204.

509 [43] E.N. Frankel, A.L. Waterhouse, P.L. Teissedre, J. Agr. Food Chem., 43 (1995)

510890 .

511 [44] L. Moreno-Barón, R. Cartas, A. Merkoçi, S. Alegret, J.M. Gutiérrez, L. Leija, 512 P.R. Hernández, R. Muñoz, M. del Valle, Anal. Lett., 38 (2005) 2189.

513 [45] J.M. Palacios-Santander, L.M. Cubillana-Aguilera, M. Cocchi, A. Ulrici, I. 514 Naranjo-Rodríguez, R. Seeber, J.L. Hidalgo-Hidalgo de Cisneros, Chemometr. Intell. 515 Lab., 91 (2008) 110.

516 [46] J.W. Tukey, Ann. Math. Stat., 29 (1958) 614.

517 [47] S.D. Peddada, 1993, p. 723. 
520 Table 1. Detailed information of the wine samples under study.

521

\begin{tabular}{cccc}
\hline Sample & Variety & $\begin{array}{c}\boldsymbol{F C} \text { Index } \\
\left(\boldsymbol{m g} \boldsymbol{L}^{-1}\right)\end{array}$ & $\begin{array}{c}\boldsymbol{I}_{280} \\
(\boldsymbol{a r b . u n i t})\end{array}$ \\
\hline S1 & Trepat & 149 & 7.53 \\
S2 & Trepat & 54 & 3.78 \\
S3 & Ull de llebre & 1199 & 27.25 \\
S4 & Picapoll negre & 933 & 31.28 \\
S5 & Marselan & 1598 & 30.64 \\
S6 & Ull de llebre & 1239 & 37.39 \\
S7 & Cabernet sauvignon & 1467 & 42.47 \\
S8 & Merlot & 1583 & 41.08 \\
S9 & Cabernet sauvignon & 1366 & 46.97 \\
S10 & Petit verdot & 1645 & 45.72 \\
S11 & Cabernet sauvignon & 1468 & 46.27 \\
S12 & Cabernet sauvignon & 1392 & 46.44 \\
S13 & Malbec & 1351 & 48.51 \\
S14 & Sumoll & 1613 & 59.61 \\
S15 & Merlot & 1358 & 47.46 \\
S16 & Merlot & 1355 & 46.10 \\
S17 & Cabernet sauvignon & 1913 & 58.58 \\
S18 & Cabernet sauvignon & 2054 & 63.73 \\
S19 & Petit verdot & 2153 & 69.80 \\
S20 & Calddoc & 2374 & 72.91 \\
\hline
\end{tabular}


523 Table 2. Fitted regression lines of the comparison between obtained vs. expected results

524 provided by the proposed ET, both for Folin-Ciocalteu and $\mathrm{I}_{280}$ indexes, averaging each

525 value per sample as pertaining to the training or external test subsets for the 25

526 replicated calculations.

527

\begin{tabular}{|c|c|c|c|c|c|c|c|c|}
\hline \multirow{5}{*}{$\begin{array}{c}\text { Train subset } \\
\text { Test subset }\end{array}$} & \multicolumn{8}{|c|}{ Folin-Ciocalteu Index } \\
\hline & \multicolumn{4}{|c|}{$A N N$} & \multicolumn{4}{|c|}{ PLS2 } \\
\hline & Correlation & Slope & Intercept & $R M S E$ & Correlation & Slope & Intercept & $R M S E$ \\
\hline & 0.998 & $0.959 \pm 0.029$ & $53.0 \pm 30.6$ & 89 & 0.999 & $1.006 \pm 0.020$ & $-11.8 \pm 3.6$ & 92 \\
\hline & 0.979 & $0.952 \pm 0.093$ & $-43.6 \pm 28.7$ & 366 & 0.932 & $1.017 \pm 0.184$ & $-149 \pm 162$ & 404 \\
\hline & \multicolumn{8}{|c|}{$I_{280}$ Index } \\
\hline & \multicolumn{4}{|c|}{$A N N$} & \multicolumn{4}{|c|}{$P L S 2$} \\
\hline & Correlation & Slope & Intercept & $R M S E$ & Correlation & Slope & Intercept & $R M S E$ \\
\hline Train subset & 0.994 & $0.965 \pm 0.049$ & $1.24 \pm 1.68$ & 3.5 & 0.995 & $0.983 \pm 0.047$ & $0.30 \pm 0.71$ & 3.8 \\
\hline Test subset & 0.963 & $0.914 \pm 0.119$ & $0.87 \pm 3.77$ & 10.1 & 0.968 & $0.974 \pm 0.118$ & $-2.64 \pm 0.69$ & 9.5 \\
\hline
\end{tabular}

528

529 


\section{$530 \quad$ Figure captions}

532 Figure 1. Some cyclic voltammograms obtained with the sensor array for some of the 533 wine samples with different Folin-Ciocalteu index: (S1) $149 \mathrm{mg} \mathrm{L}^{-1}$, (S4) $933 \mathrm{mg} \mathrm{L}^{-1}$, 534 (S11) $1468 \mathrm{mg} \mathrm{L}^{-1}$ and (S18) $2054 \mathrm{mg} \mathrm{L}^{-1}$ gallic acid equivalents. Also the signals with 535 different sensors are shown: (A) Graphite-epoxy sensor, (B) Platinum nanoparticle sensor, (C) Copper nanoparticle sensor and (D) Au microelectrode.

Figure 2. Processing scheme for building the quantification model. After optimization of the ANN, the final model has 84 approximation coefficients obtained from wavelet analysis of the sensor signals in the input layer, 10 neurons and logsig transfer function in the hidden layer and 2 neurons and purelin transfer function in the output layer.

Figure 3. Modelling ability of the optimized ANN. Comparison graph of expected vs. obtained concentrations for Folin-Ciocalteau indexes, both for (A) training and (B) testing subset. Dashed line corresponds to theoretical diagonal line.

Figure 4. Modelling ability of the optimized ANN. Comparison graph of expected vs. obtained concentrations for polyphenol indexes $\left(\mathrm{I}_{280}\right)$, both for (A) training and (B) testing subset. Dashed line corresponds to theoretical diagonal line.

Figure 5. Modelling ability of the PLS model. Comparison graph of expected vs. obtained concentrations for Folin-Ciocalteau indexes, both for (A) training and (B) testing subset. Dashed line corresponds to theoretical diagonal line.

Figure 6. Modelling ability of the PLS model. Comparison graph of expected vs. obtained concentrations for polyphenol indexes $\left(\mathrm{I}_{280}\right)$, both for $(A)$ training and $(\mathrm{B})$ testing subset. Dashed line corresponds to theoretical diagonal line. the sensor array) for some of the spiked samples. 
562 Figure 8. Score plot of the first two components obtained after PCA of the spiked 563 samples. A total of 35 samples were analysed. As can be seen, clear discrimination is 564 obtained for the different polyphenols: (I) Wine, (II) Gallic acid, (III) Catechin, (IV) $p$ 565 Coumaric acid, (V) Caffeic acid, (VI) Catechol and (VII) $m$-Cresol. 

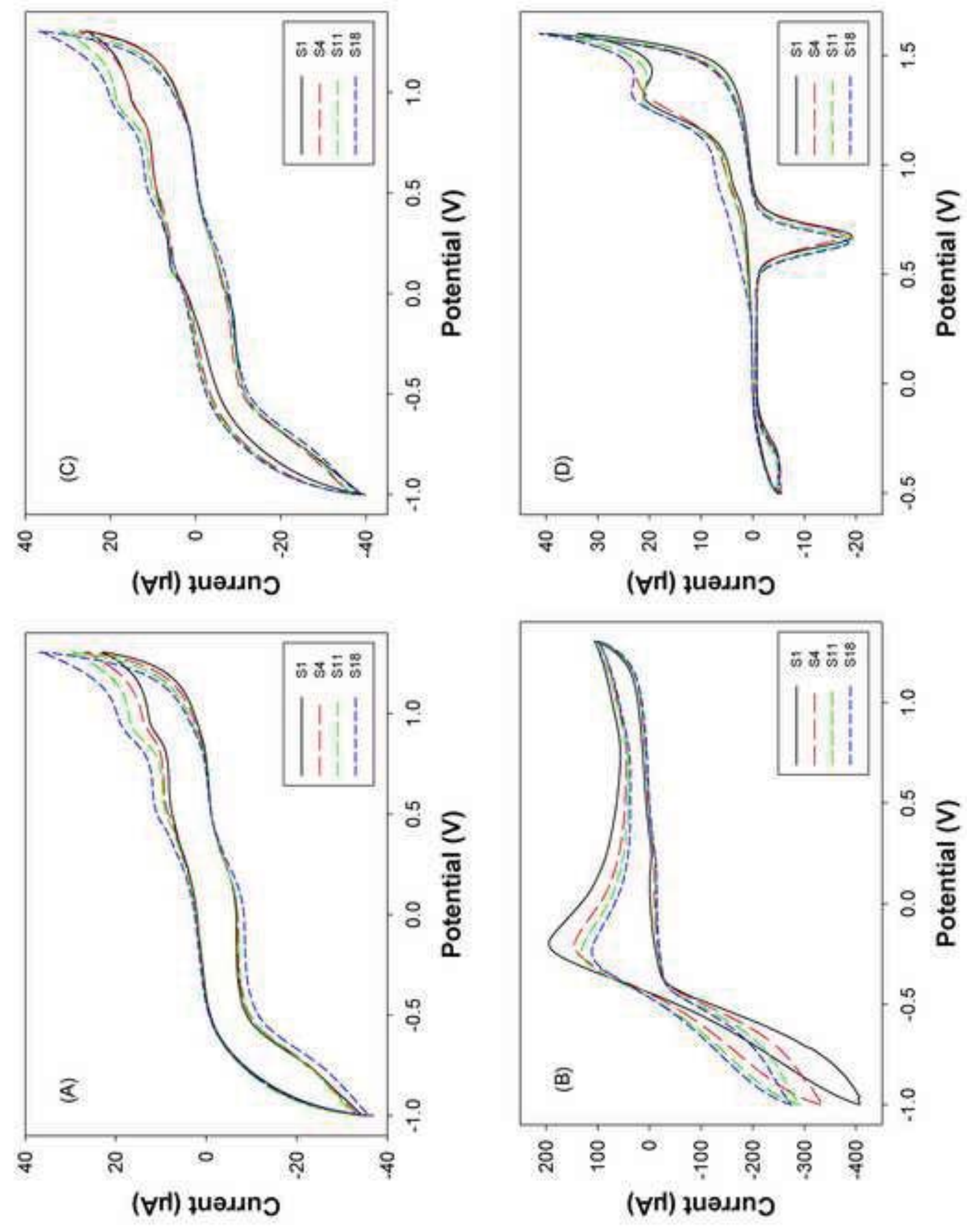


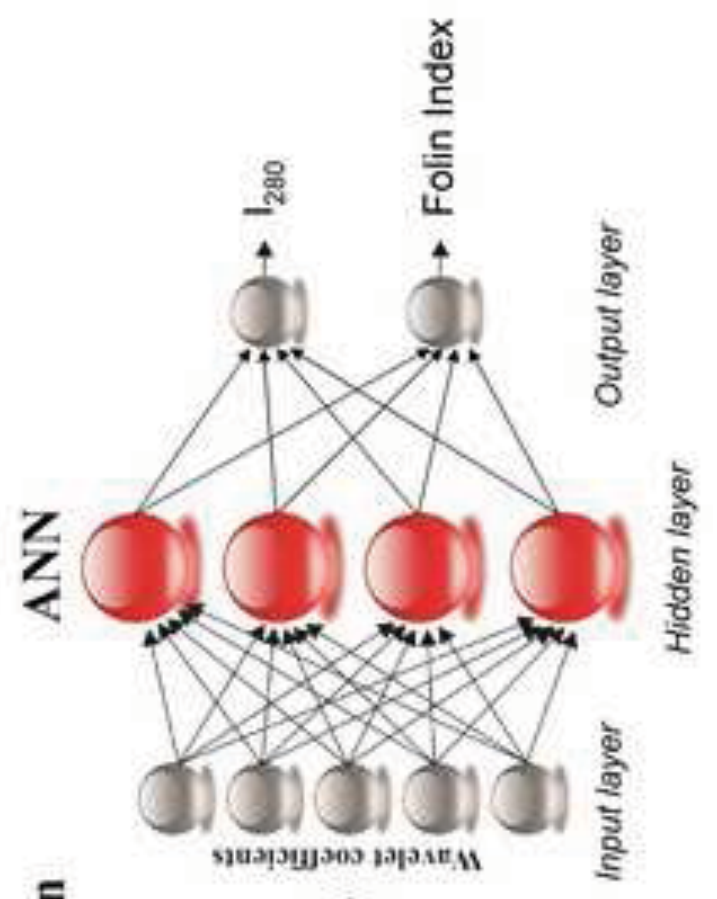

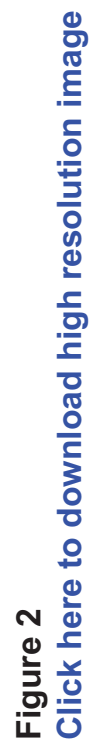

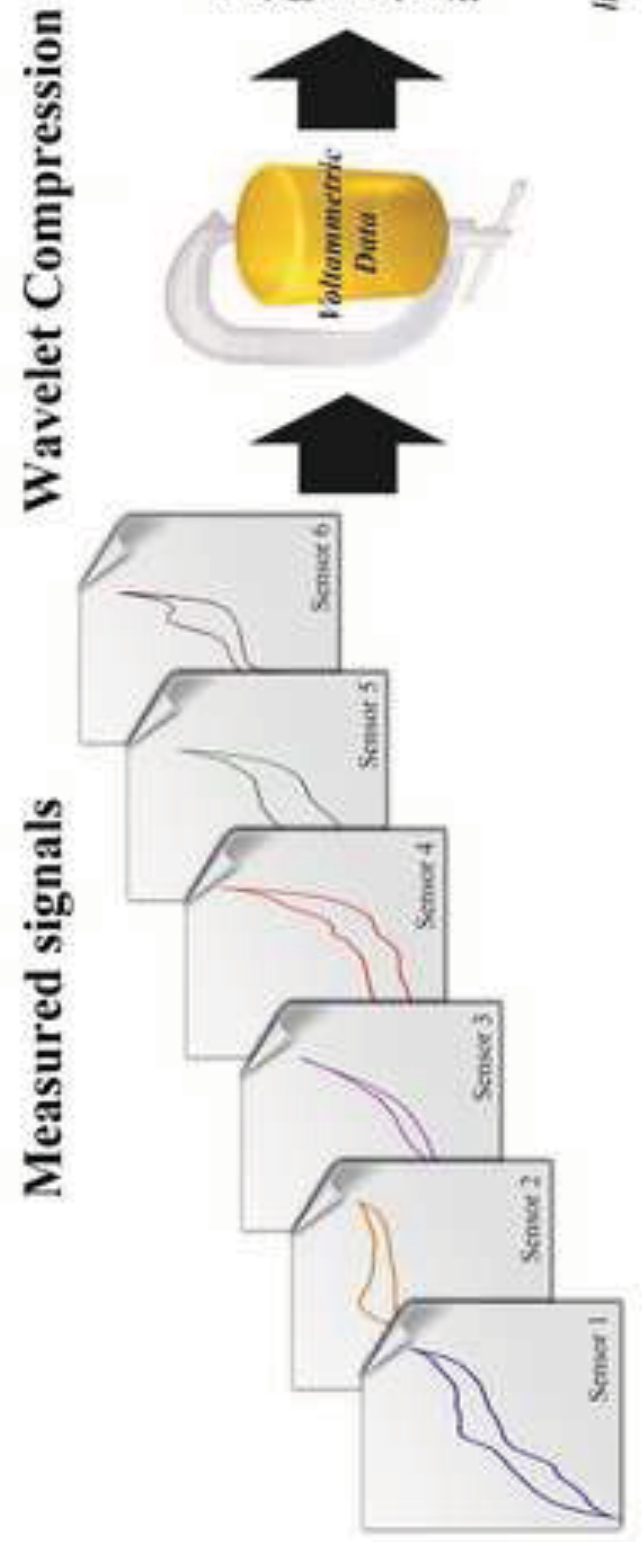




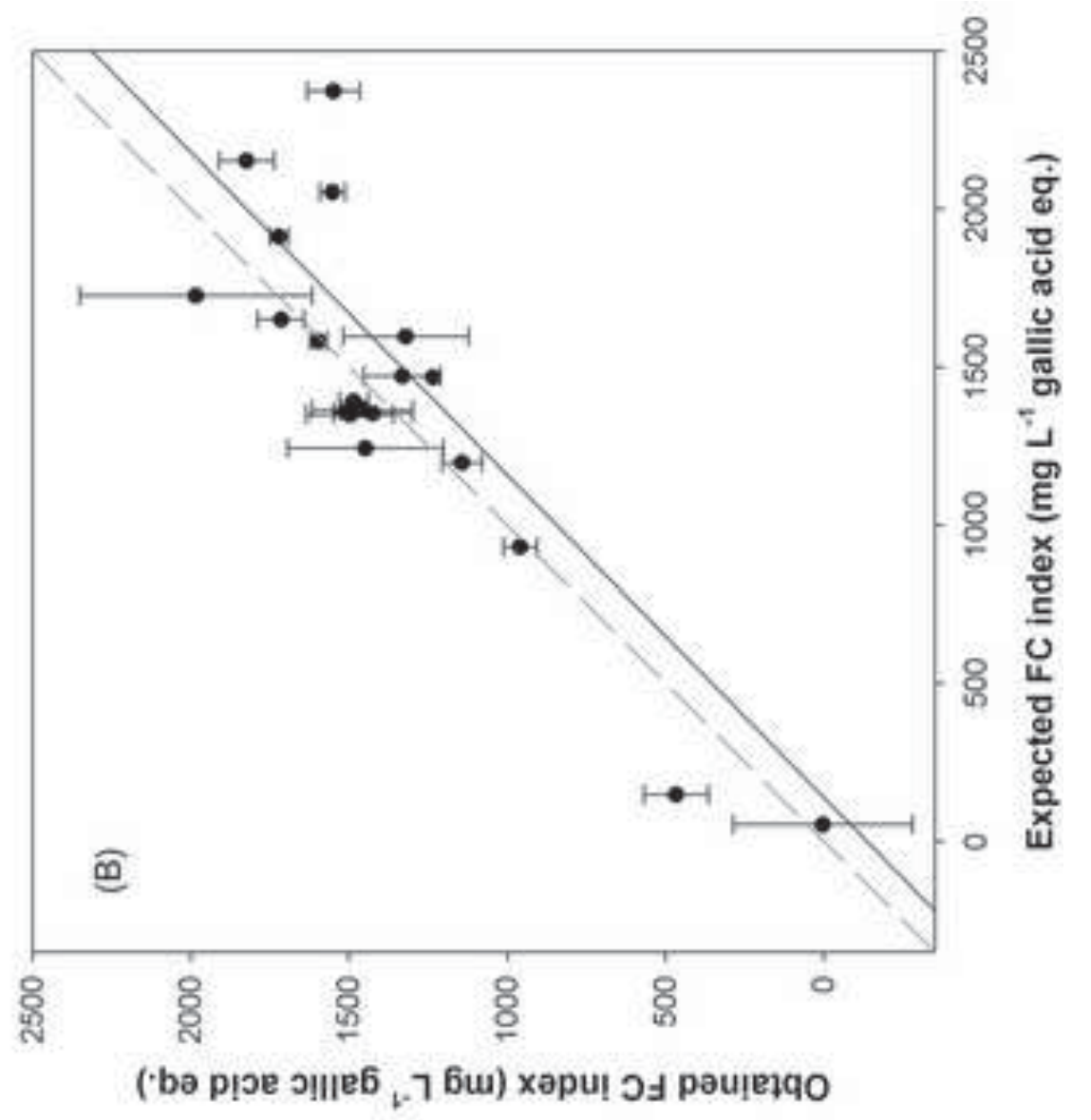

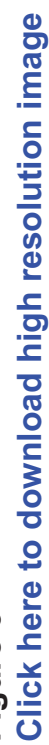

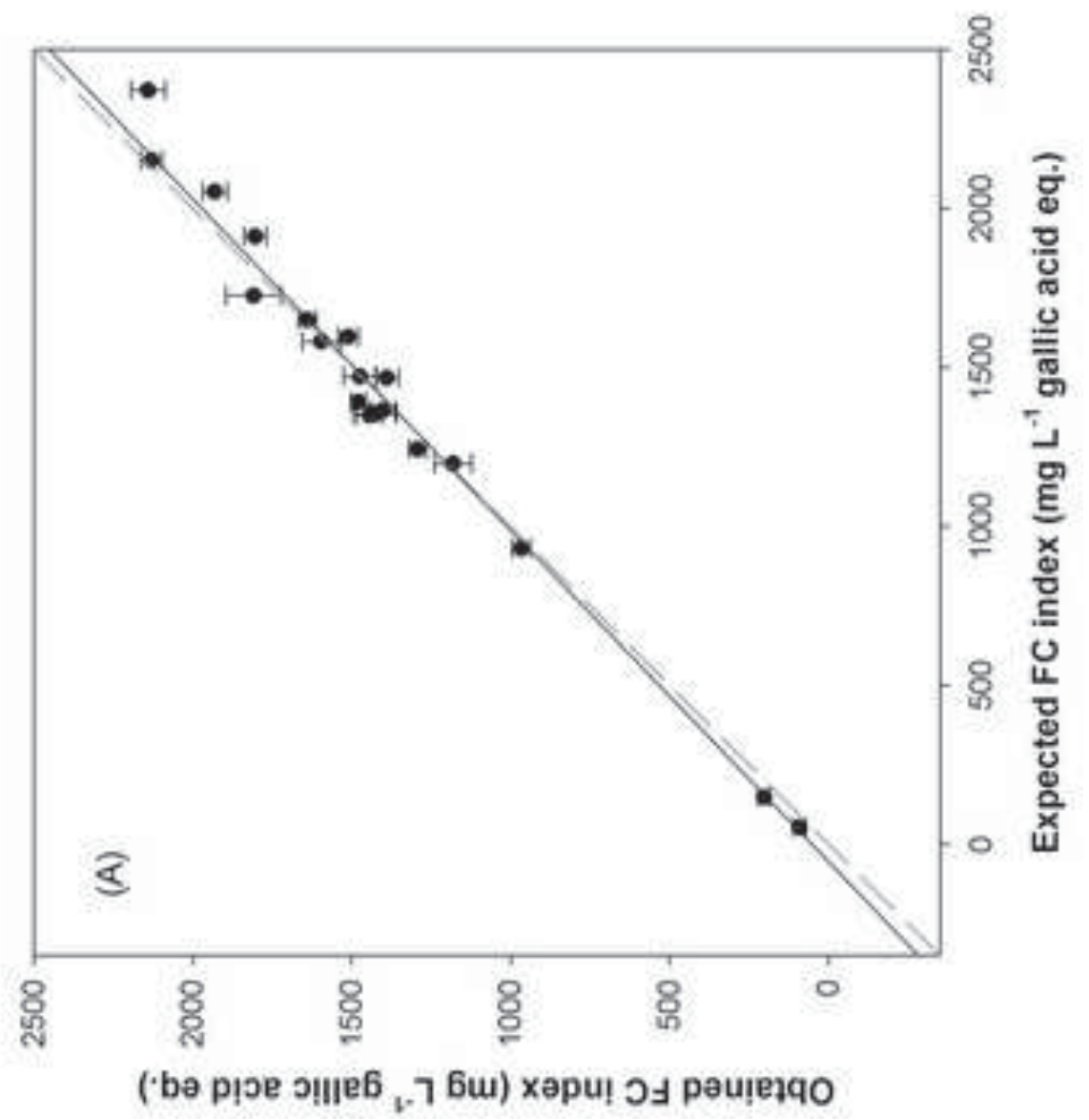



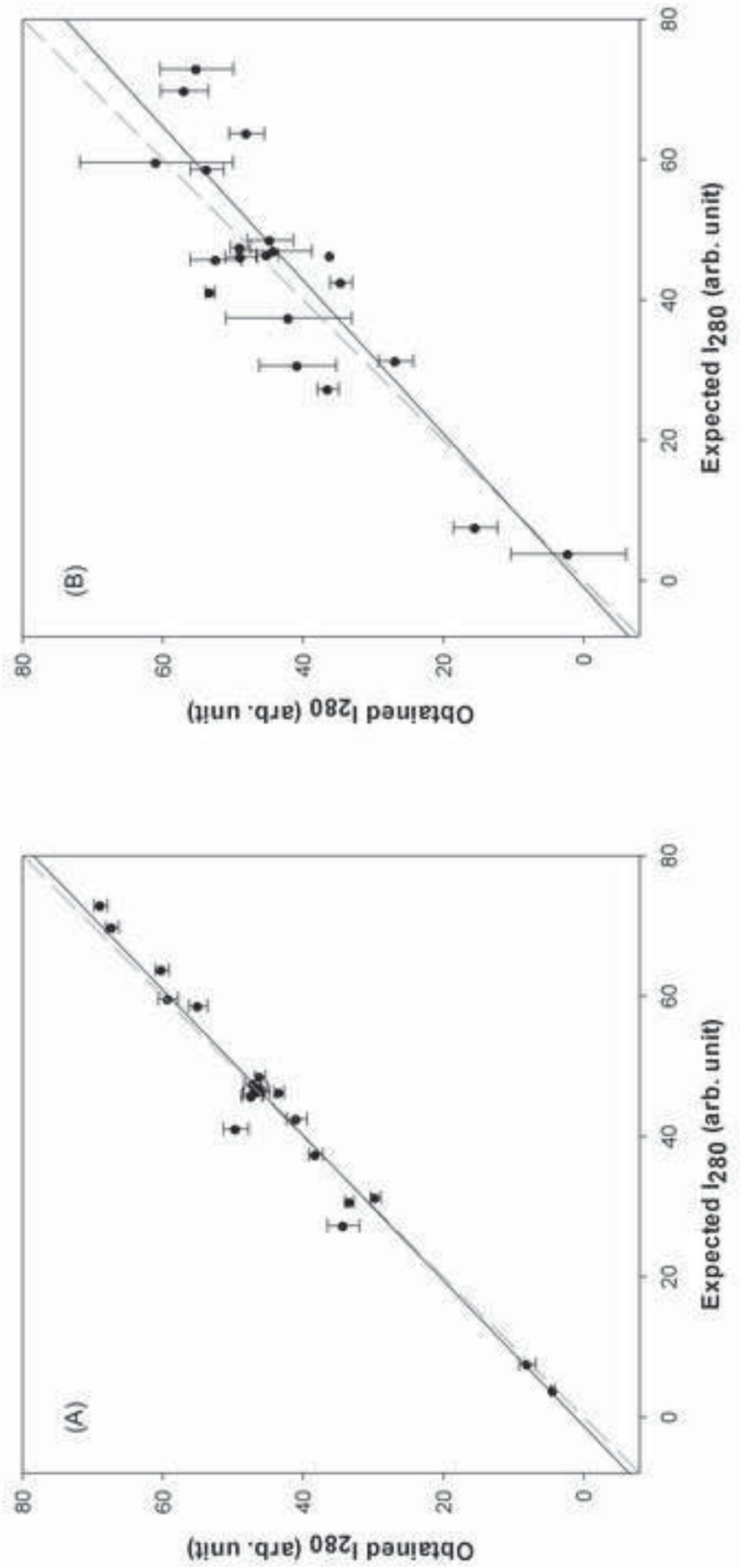

은 


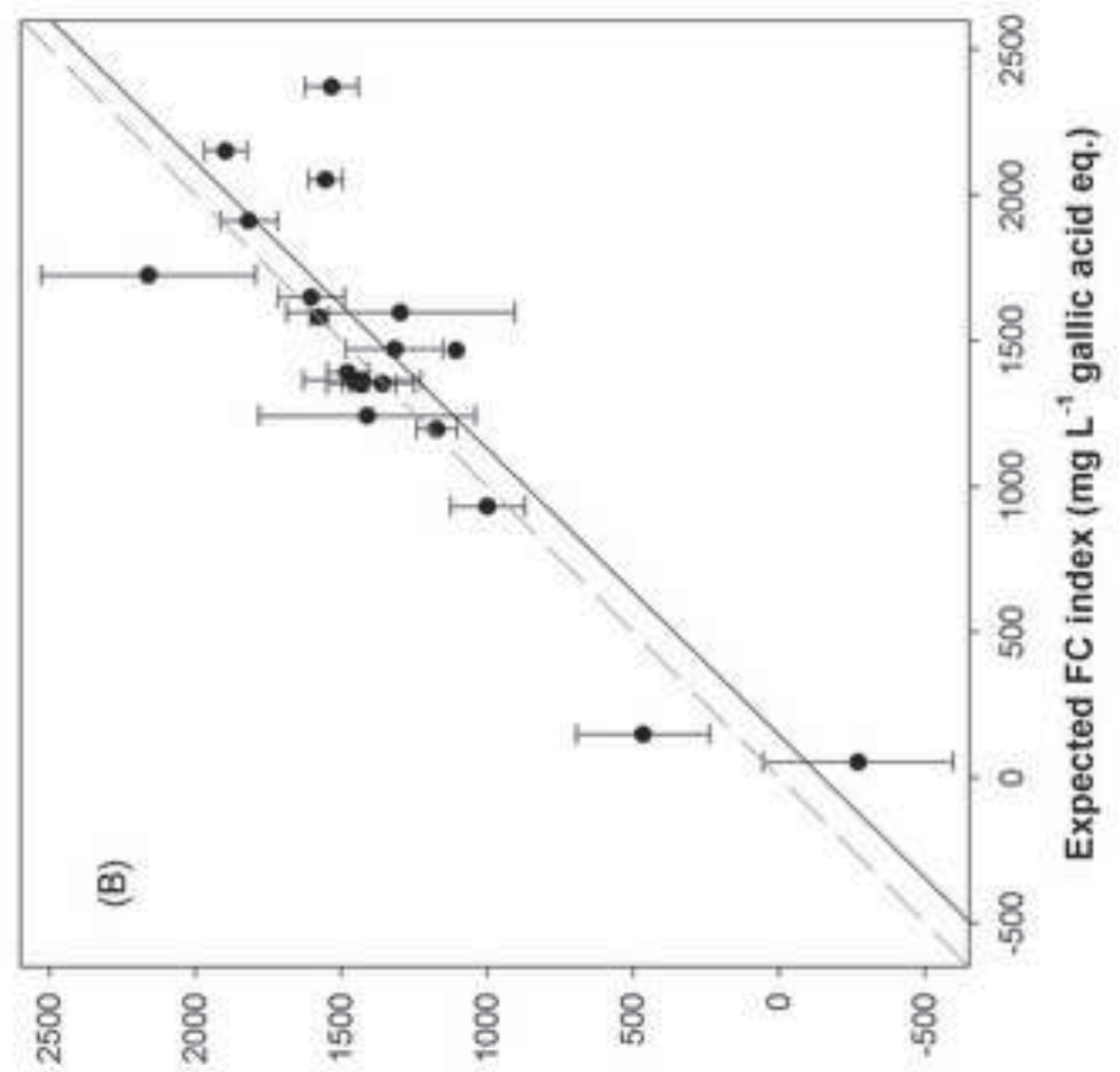

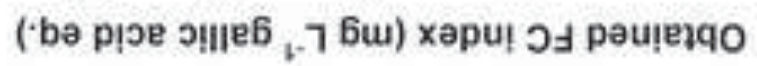

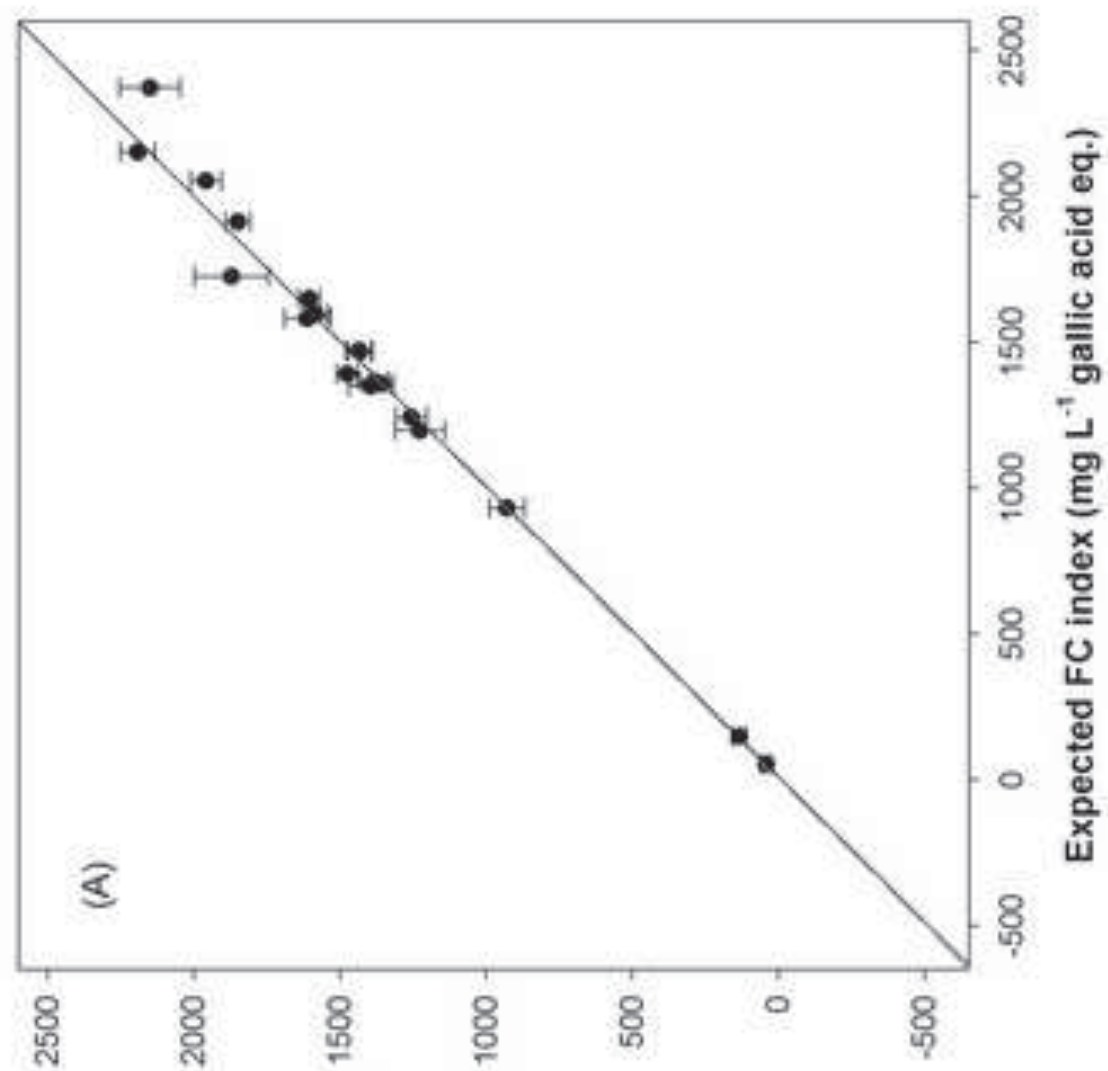

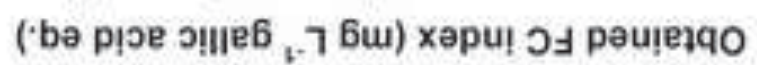



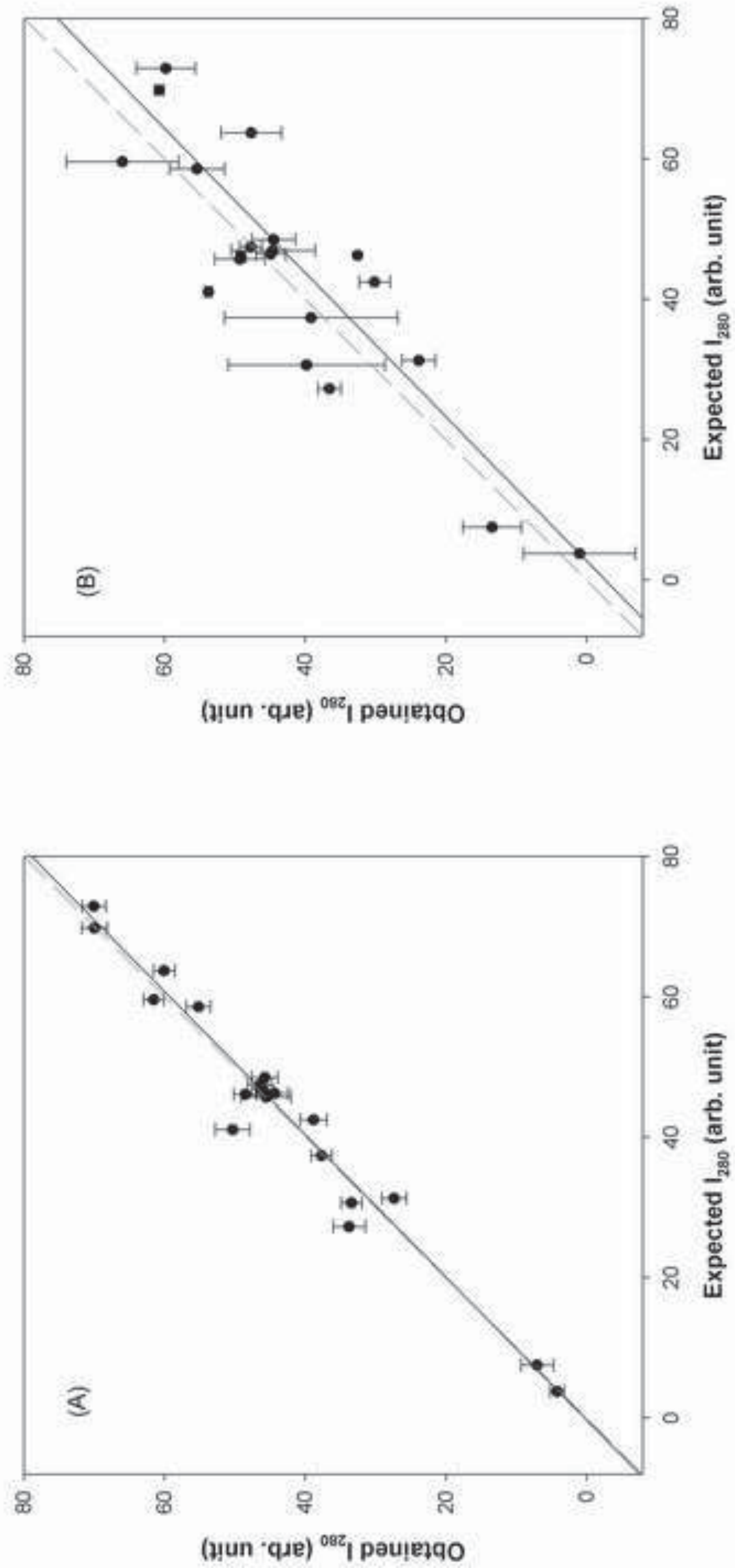


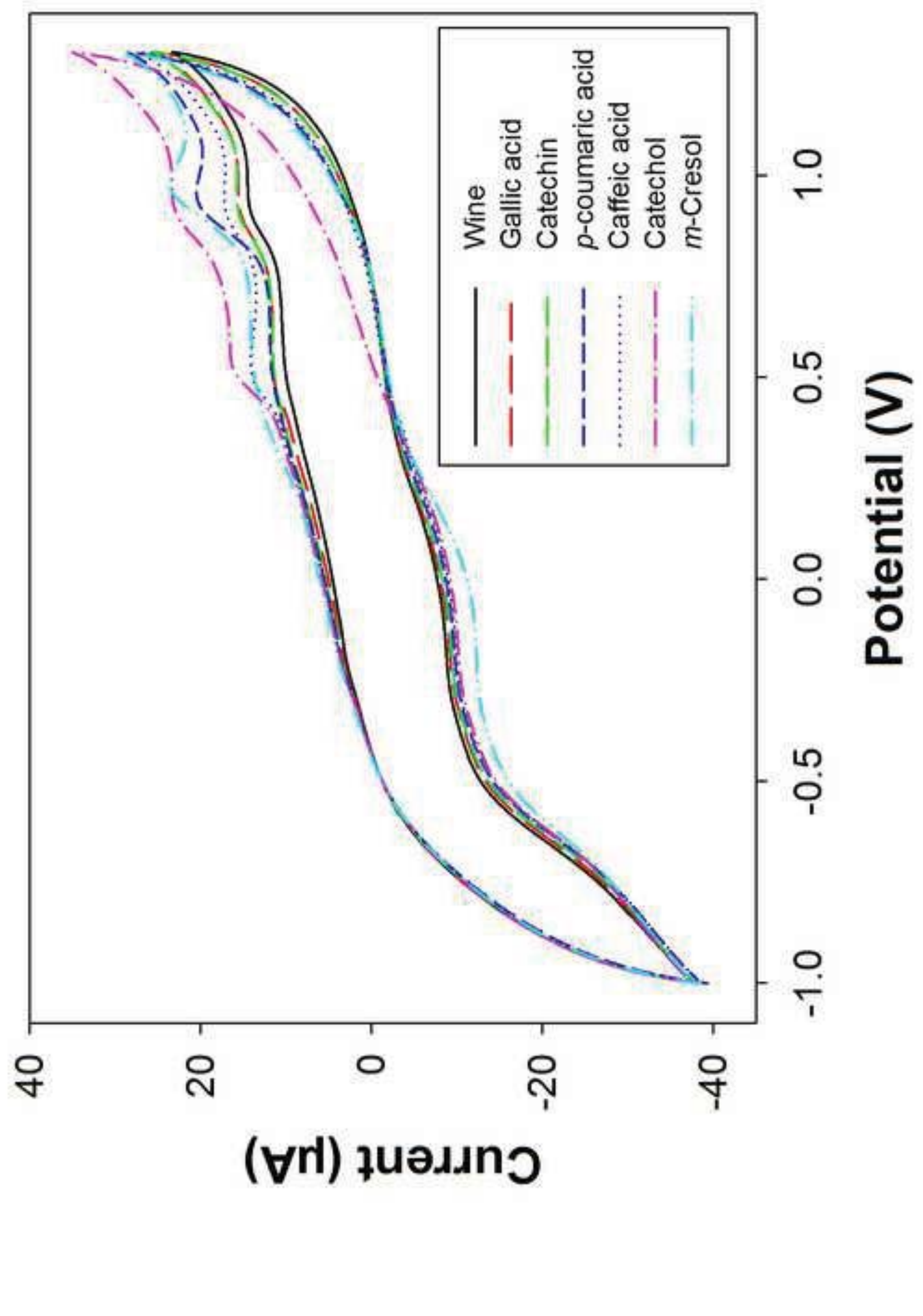




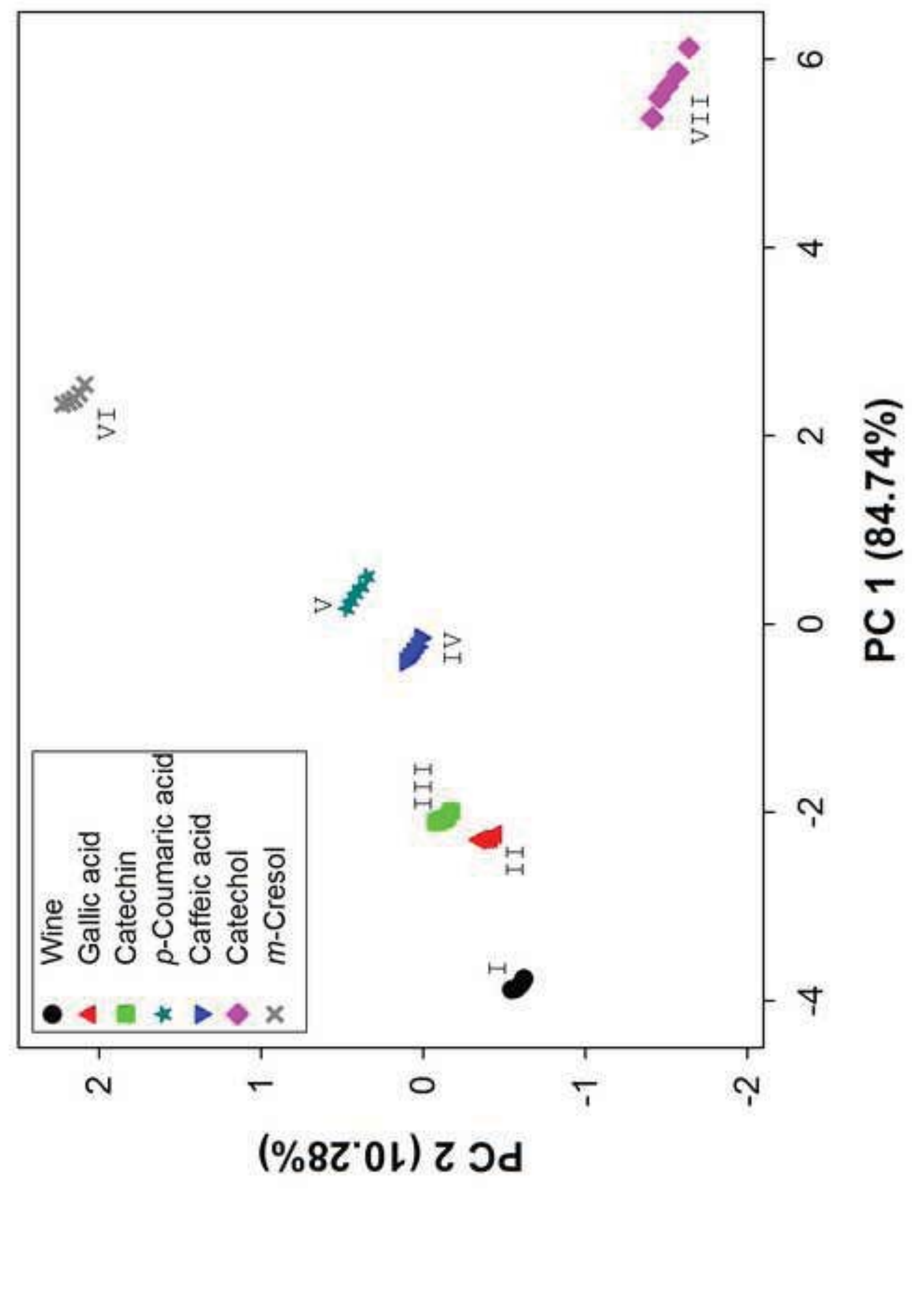

